\title{
Valores de referência do RDW-CV e do RDW-SD e sua relação com o VCM entre os pacientes atendidos no ambulatório do Hospital Universitário Oswaldo \\ Cruz - Recife, PE
}

\author{
Reference values for $R D W-C V$ and $R D W-S D$ and their relationship to mean corpuscular volume \\ among clinical patients of the Oswald Cruz Teaching Hospital, Recife, Brazil
}

\begin{abstract}
O termo anisocitose indica o grau de variação do tamanho dos eritrócitos observado ao microscópio. Essa expressão, é observada no hemograma automatizado através de dois parâmetros: $R D W-C V$ (Amplitude de Distribuição dos Eritrócitos medido como Coeficiente de Variação) e RDW-SD (o mesmo sendo medido como Desvio Padrão), que são calculados a partir do volume corpuscular médio (VCM), que representa a média do tamanho dos eritrócitos. Juntos, os dois parâmetros RDW e o VCM podem auxiliar no diagnóstico diferencial de diversas enfermidades, em especial alguns tipos de anemias. $O$ objetivo deste trabalho foi determinar os valores de referência dos parâmetros $R D W$, bem como verificar a existência de relação desses índices com o VCM a partir dos hemogramas de 306 pacientes atendidos no ambulatório do Laboratório Central do Hospital Universitário Oswaldo Cruz. Foram também determinados os valores de referência em relação ao sexo e por faixa etária. A fim de verificar a possivel relação dos parâmetros $R D W$ com VCM, foram realizados testes estatísticos de correlação de Pearson. Os valores de referência para a população geral foram semelhantes aos encontrados na literatura, enquanto que a comparação dos resultados entre sexos não apresentou diferença significativa para nenhum dos índices estudados. O estudo por faixa etária mostrou a necessidade de reavaliar a divisão das faixas etárias em estudos posteriores com esses mesmos parâmetros, pois alguns desses grupos etários não apresentaram diferença significativa entre eles. Os testes estatísticos de correlação mostraram resultados significativos para a relação entre $V C M$ microcitico e RDW-CV, bem como entre o VCM macrocitico e o RDW-SD. Para o VCM normocitico foi observada correlação significativa apenas com o $R D W-S D$ Rev. Bras. Hematol. Hemoter.
\end{abstract}

Palavras-chave: Sangue; valores de referência; eritrócitos; índice de eritrócitos.

\section{Introdução}

A amplitude de distribuição dos eritrócitos (RDW), representa, no hemograma automatizado, a presença de anisocitose ou variação do tamanho dos eritrócitos, que pode ocorrer por causas variadas, desde anemias até a presença de doenças crônicas, leucemias e uso de medicamentos. $\mathrm{O}$ índice RDW vem sendo utilizado como ferramenta complementar no diagnóstico de alguns tipos de anemias microcíticas. ${ }^{1,2}$ No entanto, pouco tem sido estudado sobre a relação do RDW com a microcitose ou macrocitose, bem como sobre a presença de elevação desses índices antes da alteração do volume corpuscular médio (VCM), que, por representar uma média do tamanho dos eritrócitos, pode

${ }^{I}$ Biomédica do Hospital Universitário Oswaldo Cruz - Universidade de Pernambuco, Recife-PE

Hospital Universitário Oswaldo Cruz - Universidade de Pernambuco, Recife-PE.

Correspondência: Leila Monteiro

Rua Vereador Olavo Viana, 95 - Bultrins

53320-330 - Olinda, PE - Brasil

E-mail:leilamonteiro3@gmail.com

Doi: 
estar normal, mesmo com anisocitose presente (RDW elevado).

Os hemogramas automatizados avaliam a média do tamanho dos eritrócitos (VCM) através da impedância, impulsos elétricos emitidos pela passagem de cada célula individualmente em um fluxo, cuja intensidade é proporcional ao tamanho das células. Esses pulsos elétricos são posteriormente, convertidos em fentolitros (fL). O RDW é então calculado como coeficiente de variação em relação à média do tamanho dos eritrócitos (VCM $)^{2,3}$ Adicionalmente, o RDW-SD é calculado pela média dos tamanhos dos eritrócitos distribuídos até $20 \%$ acima da base do histograma do eritrograma automatizado, onde se localizam todos os tamanhos de hemácias que foram avaliadas, logo, é na base do histograma que se encontra a maior variação do tamanho dos eritrócitos. ${ }^{3,4}$

Os dois índices RDW são dados matemáticos e estatísticos que contribuem de forma complementar à avaliação do VCM, por serem mais precisos e menos subjetivos quando comparados às observações microscópicas de anisocitose. ${ }^{2,4}$ Assim, valores mais baixos representam população mais homogênea que a usual, indicando extremo da normalidade, enquanto valores mais altos são a expressão numérica da anisocitose observada ao microscópio. ${ }^{2}$

Neste trabalho foram estudados pacientes atendidos no ambulatório do Hospital Universitário Oswaldo Cruz (HUOC), Recife, PE, através da consulta direta às informações constantes na requisição médica para hemograma, sendo desconsiderados os pacientes com alguma doença preexistente e os pacientes da oncologia do hospital. Foram obtidas informações sobre o sexo e a idade dos pacientes, bem como os valores de VCM, RDW-CV e RDW-SD, resultantes dos hemogramas desses pacientes, independente da presença de anemia ou não, com o objetivo de determinar os valores de referência geral, por sexo e por idade, desses índices na população atendida no serviço. Além disso, foi avaliada a relação entre VCM e o aumento ou diminuição do RDW-CV e RDW-SD, a fim de entender a utilidade diagnóstica dos indicadores automatizados de anisocitose diante da presença ou não de VCM alterado, além de tentar incentivar o uso do RDW-SD, como mais uma ferramenta guia na triagem de pacientes para o diagnóstico diferencial entre os tipos de anemias que apresentam características semelhantes com relação ao VCM.

\section{Casuística e Métodos}

A coleta dos dados foi realizada entre 306 pacientes atendidos no ambulatório do Laboratório Central do HUOC com solicitação médica para realização de hemograma. As informações como sexo e idade foram obtidas diretamente pela consulta à requisição médica contendo o nome e a data de nascimento dos pacientes. Todos os pacientes provenientes do setor de oncologia do hospital foram excluídos.
Os eritrogramas foram realizados utilizando-se equipamento automatizado Sysmex XT-1800, da Roche Diagnóstica ${ }^{\circledR}$. A partir dos hemogramas automatizados, foram obtidos os resultados de RDW-SD, RDW-CV e VCM de cada paciente.

Os valores de referência geral, por sexo e por idade, foram determinados a partir de pacientes considerados normais para cada um dos índices estudados com base nos limites preconizados para esses parâmetros nos aparelhos da linha Sysmex - Roche Diagnóstica ${ }^{\circledR}$ : VCM 80-100fL; RDWCV 12 a 14,4\% e RDW-SD 38,6 a 49,1fL. ${ }^{3}$ (Disponível em: http://www.rochediagnostica.com.br/lab_system/web/ artigos_hematologia2.asp. Acesso em 24/05/2008.). Os dados foram tratados estatisticamente para obtenção da média e desvios padrões de cada índice.

Com relação à idade, os pacientes foram divididos nas seguintes faixas etárias: menores de 6 anos, 7 a 10 anos, 11 a 17 anos, 18 a 59 anos e maiores de 60 anos. Os valores encontrados para cada um desses grupos etários foram ainda comparados através de testes estatísticos de significância, para avaliar e importância dessa divisão etária.

Com o objetivo de avaliar a possível relação existente entre o VCM e cada um dos índices RDW, foram comparados três grupos de VCM: macrocítico (VCM > $100 \mathrm{fL}$ ), normocítico (VCM entre 80 e $100 \mathrm{fL}$ ) e microcítico (VCM $<80 \mathrm{fL}$ ), contra as variações existentes para RDW-SD e RDW-CV, segundo os intervalos de referência acima mencionados.

A análise estatística dos dados foi realizada através do programa Microsoft Excel 2003, utilizando-se o teste de correlação de Pearson para a comparação entre os índices em cada um dos três grupos de VCM e T student como teste de significância. $\mathrm{O}$ teste $\mathrm{F}$ foi utilizado para comparar os resultados entre os sexos e entre as faixas etárias.

\section{Resultados e Discussão}

Valores de referência geral e em relação ao sexo Os valores de referência encontrados para a população geral mostraram que não houve diferença significativa entre estes e os observados pela literatura consultada (Tabela 1). Bain afirma que os valores de referência para o VCM e RDWCV são respectivamente, 82 a 98 fL e 11,6 a 13,9 (considerando, para este último, o aparelho Technicon S Plus, de meto-

Tabela 1. Valores de referência geral na população atendida no ambulatório do Laboratório Central do Hospital Universitário Oswaldo Cruz

\begin{tabular}{lccc}
\hline $\begin{array}{l}\text { Valores de } \\
\text { Referência }\end{array}$ & VCM (fL) & RDW-CV (\%) & RDW-SD (fL) \\
\hline $\begin{array}{l}\text { População } \\
\text { Estudada }\end{array}$ & $80,83-99,19^{*}$ & $12,2-14,5^{*}$ & $38,3-48,9^{*}$ \\
Literatura & $80-98^{\star *}$ & $11-14,5^{\star \star}$ & $38,6-49,1^{\star \star \star}$ \\
\hline
\end{tabular}

* Valores representados pela média \pm 2 desvios padrões

${ }^{* *}$ Failace. ${ }^{2 * * * * *}$ Villas Boas, et al. $(2003)^{3}$ 
Tabela 2. Valores de referência por sexo entre a população atendida no ambulatório do Laboratório Central do Hospital Universitário Oswaldo Cruz

\begin{tabular}{cccc}
\hline & VCM (fL) & RDW-CV (\%) & RDW-SD (fL) \\
\hline Feminino & $82,2-97,1$ & $12,4-15,8$ & $40,0-49,9$ \\
& $(1,06)^{* a}$ & $(1,02)^{* \mathrm{~b}}$ & $(0,92)^{* \mathrm{c}}$ \\
Masculino & $83,1-100,3$ & $12,4-15,8$ & $40,1-51,6$ \\
& $(1,06)^{\mathrm{a}}$ & $(1,02)^{\mathrm{b}}$ & $(0,92)^{\mathrm{c}}$ \\
Literatura & $80-98^{* *}$ & $11-14,5^{* *}$ & $38,6-49,1^{* * *}$ \\
\hline
\end{tabular}

*Valores representados pela média \pm 2 desvios padrões. ${ }^{* *}$ Failace ${ }^{2 ;}{ }^{* * * * * *}$ Villas Boas, et al. ${ }^{3}$; a: valor de $\mathrm{F}$ no teste de significância entre os sexos para VCM $(F c=1,35)$; b: valor de $F$ no teste de significância entre os sexos para RDW-CV $(F c=1,38)$; c: valor de $F$ no teste de significância entre os sexos para RDW-SD $(F c=0,73)$. Se $F>F c=$ Há diferença significativa entre os sexos; Se $\mathrm{F}<\mathrm{Fc}=$ Não há diferença significativa entre os sexos.

Tabela 3. Valores de referência por faixas etárias entre a população atendida no ambulatório do Laboratório Central do Hospital Universitário Oswaldo Cruz

\begin{tabular}{lccc}
\hline & VCM (fL) & RDW-CV (\%) & RDW-SD (fL) \\
\hline$\leq 6$ anos* & $74,9-86,3$ & $12,7-16,5$ & $36,7-49,2$ \\
$7-10$ anos & $81,5-91,2$ & $13,1-13,8$ & $38,1-44,3$ \\
$11-17$ anos & $85,3-95,4$ & $12,3-13,9$ & $40-44,9$ \\
18 a 59 anos & $81,7-98,8$ & $12,3-15,8$ & $39,7-50,5$ \\
$\geq 60$ anos & $79,1-105,5$ & $10,7-17,4$ & $36-57,1$ \\
\hline
\end{tabular}

* Valores representados pela média \pm 2 desvios padrões dologia semelhante à utilizada pelo Sysmex). ${ }^{5}$ Adicionalmente, Failace menciona os valores de referência do RDW-CV para o Coulter (11 a 14,5) e Cell Dyn 4000 (12 a 15), ambos com tecnologia similar ao do equipamento utilizado no presente trabalho. O mesmo autor também considera a faixa de normalidade para o VCM entre 80 a 98 fL. ${ }^{2}$ Esses valores são bastante próximos aos encontrados no presente trabalho e ao observado por Villas-Boas (2003) utilizando os aparelhos da linha Sysmex.

Com relação aos sexos não houve diferença estatística entre eles para o VCM, bem como para os dois índices RDW (Tabela 2).

\section{Valores de referência por faixa etária}

$\mathrm{Na}$ tabela 3 podem ser visualizados os valores de referência por faixa etária. Além disso, foi realizado ainda o estudo estatístico (teste F), comparando esses dados entre as faixas etárias previamente determinadas. Foi observado que, com relação ao VCM, o grupo de menores de 6 anos, 18 a 59 anos e maiores de 60 anos apresentaram diferença significativa com todos os demais, enquanto os grupos de 7 a 10 anos e 11 a 17 anos não obtiveram resultados significativos entre si (Tabela 4).

Por outro lado, quando comparados os valores do RDW-CV, observou-se que as faixas etárias de menores de 6 anos e de 7 a 10 anos tiveram resultados significativamente diferentes entre si, enquanto os de idade entre 18 a 59 anos e maiores de 60 anos não apresentaram diferença significativa entre si e sim com a faixa entre 11 a 17 anos, a qual também foi diferente estatisticamente do grupo de 7 a 10 anos (Tabela 5).

No entanto, os valores de RDWSD não apresentaram diferença significativa entre as duas faixas etárias mais jovens, nem entre os grupos de 11 a 17 anos e 18 a 59 anos, tendo sido encontrada diferença estatistica entre os valores de referência das faixas etárias menores de 6 anos e maiores de 60 anos (Tabela 6).

Em estudo realizado com 184 crianças entre 0 a 5 anos de idade, Almeida et al. ${ }^{6}$ encontraram valores médios de $79,5 \mathrm{fL}$ e $14,9 \%$, respectivamente, para VCM e RDW-CV. Esses valores se mostraram próximos aos encontrados no presente trabalho. Por outro lado, no caso dos adolescentes (faixa entre 11 a 17 anos), os limites de referência estudados se mostraram superiores aos encontrados por Silva et $a l .^{7}$ entre pacientes não anêmicos com idade entre 10 e 20 anos em estudo realizado na Universidade Federal de
Resultados representados por: teste $\mathrm{F}$ (Fcrítico). $\mathrm{Se} F>\mathrm{Fc}=$ Há diferença significativa

entre as faixas etárias; $\mathrm{Se} F<F c=$ Não há diferença significativa entre as faixas etárias
Tabela 5. Correlação dos resultados de RDW-CV entre as faixas etárias estudadas*

\begin{tabular}{lccccc}
\hline$\leq$ de 6 anos & - & $0,52(0,19)$ & $2,67(4,95)$ & $1,38(4,40)$ & $1,36(4,41)$ \\
7 a 10 anos & $0,52(0,19)$ & - & $5,06(4,95)$ & $2,63(4,40)$ & $2,58(4,41)$ \\
11 a 17 anos & $2,67(4,95)$ & $5,06(4,95)$ & - & $0,51(0,45)$ & $0,50(0,44)$ \\
18 a 59 anos & $1,38(4,40)$ & $2,63(4,40)$ & $0,51(0,45)$ & - & $1,01(1,43)$ \\
$\geq$ de 60 anos & $1,36(4,41)$ & $2,58(4,41)$ & $0,50(0,44)$ & $1,01(1,43)$ & - \\
\hline
\end{tabular}


Tabela 6. Correlação dos resultados de RDW-SD entre as faixas etárias estudadas*.

\begin{tabular}{lccccc}
\hline & $\leq$ de 6 anos & 7 a 10 anos & 11 a 17 anos & 18 a 59 anos & $\geq$ de 60 anos \\
\hline$\leq$ de 6 anos & - & $1,20(4,87)$ & $1,84(4,20)$ & $0,65(0,48)$ & $0,50(0,47)$ \\
7 a 10 anos & $1,20(4,87)$ & - & $0,65(0,22)$ & $0,78(0,43)$ & $0,60(0,43)$ \\
11 a 17 anos & $1,84(4,20)$ & $0,65(0,22)$ & - & $1,20(3,70)$ & $0,93(0,45)$ \\
18 a 59 anos & $0,65(0,48)$ & $0,78(0,43)$ & $1,20(3,70)$ & - & $1,29(1,34)$ \\
$\geq$ de 60 anos & $0,50(0,47)$ & $0,60(0,43)$ & $0,93(0,45)$ & $1,29(1,34)$ & - \\
\hline
\end{tabular}

${ }^{*}$ Resultados representados por: teste $\mathrm{F}$ (Fcrítico). Se F>Fc = Há diferença significativa entre as faixas etárias; $\mathrm{Se} F<\mathrm{Fc}=$ Não há diferença significativa entre as faixas etárias

igualam os percentuais de homens e mulheres na população estudada.

Além disso, em 1976, Proctor et al. ${ }^{10}$ obtiveram valores de VCM usando material de controle de qualidade para determinar os limites de referência para este parâmetro no aparelho Coulter Counter e encontraram os seguintes valores: 84 a 94 fL. Resultados semelhantes foram encontrados por Davidson e Hamilton, ${ }^{11}$ que observaram os valo-

Tabela 7. Correlação entre os indices VCM, RDW-CV e RDW-SD no grupo microcítico $(V C M<80 \mathrm{fL})$

\begin{tabular}{ccc}
\hline & RDW-CV* & RDW-SD \\
\hline VCM & $-0,31(\mathrm{~S})^{* *}$ & $0,25(\mathrm{~S})$ \\
RDW-CV & - & $0,80(\mathrm{NS})$ \\
RDW-SD & $0,80(\mathrm{NS})$ & - \\
\hline
\end{tabular}

Resultados representados por: índice de correlação R: $R>0$, correlação positiva; $R<0$, correlação negativa; $R=0$ ou próximo de zero até $\pm 0,10$, sem correlação. ${ }^{* *} S=$ resultados significativos pelo teste $\mathrm{T} ; \mathrm{NS}=$ resultados não significativos pelo teste $\mathrm{T}$

Tabela 8. Correlação entre os índices VCM, RDW-CV e RDW-SD no grupo microcítico (VCM > 100fL)

\begin{tabular}{ccc}
\hline & RDW-CV & RDW-SD \\
\hline VCM & $-0,09(\mathrm{~S})$ & $0,34(\mathrm{~S})$ \\
RDW-CV & - & 0,87 (NS) \\
RDW-SD & 0,87 (NS) & -
\end{tabular}

Resultados representados por: índice de correlação R: R>0, correlação positiva; $R<0$, correlação negativa; $R=0$ ou próximo de zero até $\pm 0,10$, sem correlação. ${ }^{*} S=$ resultados significativos pelo teste $\mathrm{T} ; \mathrm{NS}=$ resultados não significativos pelo teste $\mathrm{T}$

São Paulo, que foi de 73,2 a 95,76fL (média \pm 2 desvios padrões).

Segundo Macedo (2002) in Barbosa et al. ${ }^{8}$ os valores de referência para o VCM em pessoas maiores de 60 anos é de 81 a 99fL e de 80 a $98 \mathrm{fL}$ para o sexo feminino e masculino, respectivamente, enquanto o RDW-CV é de $10 \%$ a $15 \%$ para ambos os sexos, sendo esses valores semelhantes aos encontrados no nosso trabalho para a faixa etária acima referida.

Por outro lado, em trabalho realizado por Costa et al., ${ }^{9}$ que comparou pacientes controles (sem doença hepática) e doentes (com doença hepática) com idade entre 35 a 74 anos, foram encontrados, no grupo controle, valores de referência para VCM e RDW-CV, respectivamente de 82,67 a 94,27fLe 13,61 a $14,73 \%$. Esses valores se mostraram mais próximos daqueles encontrados para a faixa etária entre 18 a 59 anos aqui estudada, porém foram diferentes para o nosso grupo de pacientes acima de 60 anos. Tal observação pode ser explicada pela diferença na divisão da faixa etária e pela presença de um percentual muito maior de homens no estudo de Costa et al. ${ }^{9}$ do que no nosso, onde praticamente se res de VCM em pacientes com 15 a 69 anos com intervalo de referência entre 80 a 99fL. Neste mesmo trabalho, o VCM no sexo feminino foi de 81,2 a 96,2 (média \pm 2 desvios padrões), enquanto no masculino foi de 81,02 a 95,42 (média \pm 2 desvios padrões). Tais resultados foram bastante semelhantes aos aqui expostos, tanto para o valor de referência geral $(80,83$ a $99,19 \mathrm{fL})$ quanto com relação aos limites para os sexos, especialmente o feminino ( 82,2 a $97,1 \mathrm{fL})$.

\section{Correlação entre os parâmetros RDW e VCM}

No que diz respeito aos testes de correlação, no grupo microcítico ( $\mathrm{n}=21$ pacientes) foi observado que a maior parte $(59,0 \%)$ apresentou resultados de RDW-CV acima do limite superior de normalidade $(14,4 \%)$.

$\mathrm{O}$ teste de correlação do VCM versus RDW-CV mostrou que valores menores de VCM se correlacionam com valores maiores de RDW-CV (significativos). Quando comparado com o RDW-SD, o grupo microcítico apresentou baixa correlação com o VCM (porém significativa), enquanto na comparação entre os dois índices RDW foi observada correlação positiva, isto é, aumentos de um índice, correspondendo à elevação nos valores do outro, porém não significativa (Tabela 7).

No grupo macrocítico ( $\mathrm{n}=28$ pacientes), 78,5\% apresentaram RDW-SD acima dos limites de referência, enquanto $39,28 \%$ tinham RDW-CV maior que $14,4 \%$. A avaliação estatística dos resultados acima mostrou que não houve correlação do VCM macrocítico frente ao RDW-CV, enquanto com o RDW-SD foi observado que aumentos nos valores do VCM correspondem a elevações do RDW-SD (significativos). No entanto, o RDW-CV e o RDW-SD apresentaram correlação positiva entre si, porém estatisticamente não significativa (Tabela 8).

O grupo normocítico foi o que apresentou maior quantidade de pacientes $(\mathrm{n}=256)$, no entanto apresentou correlação apenas na comparação entre os valores de RDW$\mathrm{SD}$ e o VCM. O teste de correlação entre os dois índices RDW, apesar de ter sido positiva, não apresentou diferença significativa no teste $\mathrm{F}$, enquanto no caso do RDW-CV frente ao VCM normocítico houve correlação baixa, porém significativa (Tabela 9). Por outro lado, do total de 256 pacientes com normocitose, $22,65 \%$ apresentaram RDW-CV maior 
Tabela 9. Correlação entre os índices VCM, RDW-CV e RDW-SD no grupo normocítico (VCM entre 80 a 100fL)

\begin{tabular}{ccc}
\hline & RDW-CV* & RDW-SD \\
\hline VCM & $-0,17(\mathrm{~S})$ & $0,27(\mathrm{~S})$ \\
RDW-CV & - & $0,88(\mathrm{NS})$ \\
RDW-SD & $0,88(\mathrm{NS})$ & - \\
\hline
\end{tabular}

Resultados representados por: índice de correlação R: $R>0$, correlação positiva; $R<0$, correlação negativa; $R=0$ ou próximo de zero até $\pm 0,10$, sem correlação. ${ }^{* \star} S=$ resultados significativos pelo teste $\mathrm{T} ; \mathrm{NS}=$ resultados não significativos pelo teste $\mathrm{T}$

que $14,4 \%$ e $12,5 \%$ deste grupo tiveram RDW-SD maior que 49,1fL.

Com base nos resultados acima expostos, no grupo microcítico foi observada uma relação significativa entre o VCM e o RDW-CV, o que confirma o resultado encontrado por Villas Boas et al., ${ }^{3}$ que concluíram que este índice é o mais sensível para indicação da heterogeneidade da população de eritrócitos nos casos de microcitose, diferentemente do RDW-SD, o qual não se mostrou sensível nesses casos. Resultados semelhantes foram observados por Ferreira, ${ }^{12}$ onde os pacientes com microcitose e hipocromia apresentaram valores maiores de RDW-CV quando comparados com os demais grupos estudados (normocítico/normocrômico, microcítico/normocrômico e macrocítico).

Por outro lado, no presente trabalho, o grupo macrocítico apresentou correlação significativa apenas com o RDW-SD, da mesma forma que o encontrado no estudo de Ferreira, ${ }^{12}$ no qual foram encontrados valores médios deste índice no grupo macrocítico, superiores aos dos demais grupos estudados pelo autor.

Adicionalmente, Villas Boas et al. ${ }^{3}$ também concluíram que o RDW-SD apresenta vantagens na detecção de anisocitose nos casos de VCM maior que 100fL, sendo mais sensível que o RDW-CV na representação da heterogeneidade da população eritroide neste grupo. Isto pode ser justificado pelo fato do RDW-CV ser calculado pela relação entre o desvio padrão da média de distribuição por tamanho e o valor do VCM. Assim, quando o VCM está elevado, o valor do desvio é mascarado pelo do VCM no cálculo do RDW-CV. Este fato não acontece com o RDW-SD, que é determinado pela variação do tamanho em fentolitros na população compreendida até $20 \%$ acima da base do histograma automatizado. $^{3,4}$

Em contraste com esses resultados, Matos et al. ${ }^{13}$ afirmaram em seu estudo com pacientes com anemia ferropriva, anemia de doença crônica e ß-talassemia que o RDWCV não foi útil na diferenciação entre esses tipos de enfermidades. No entanto, o mesmo admite e explica que existem outros trabalhos afirmando a utilidade desses índices para este fim. Aslan et al., ${ }^{14}$ por exemplo, observaram que o RDW$\mathrm{CV}$ apresentou valores superiores entre os casos de pacientes em curso de terapia com ferro, sendo este índice, portanto, útil na diferenciação de anemia ferropriva em tratamento de outras anemias microcíticas e hipocrômicas, como a ß- talassemia. Os autores observaram, ainda que esse aumento do RDW-CV coincide com a reticulocitose que ocorre com o tratamento, sendo que, no entanto o primeiro permanece alterado por mais tempo que o segundo.

Ainda no que diz respeito à $\beta$-talassemia, Akai et al. ${ }^{15}$ observaram que o RDW-SD foi mais útil na distinção entre esse tipo de anemia com a anemia ferropriva, pois este índice se mostrou inferior a $32 \mathrm{fL}$ no primeiro caso, havendo, portanto homogeneidade na população de eritrócitos em comparação com a anemia ferropriva, que normalmente é acompanhada de anisocitose. Este fato é explicado pela coexistência de eritrócitos no sangue periférico em estágios progressivos de deficiência de ferro, existindo uma mistura de normócitos com micrócitos no sangue periférico. ${ }^{16}$

Da mesma forma, Bain afirma que a primeira evidência de deficiência de ferro seria o aumento do RDW, o qual precede a anemia, enquanto, comparativamente, este índice se mantém dentro da normalidade nos casos de $\beta$-talassemia e de anemia de doença crônica. ${ }^{17}$

O grupo normocítico aqui estudado, apresentou casos isolados de anisocitose por elevação de RDW-CV ou SD sem alteração do VCM, podendo esse fato ser explicado pela presença de anemia ferropriva ou macrocítica em fase inicial, anemias sidroblásticas ou leucemia linfoblástica crônica, como mencionado por Almeida et al. ${ }^{1}$ Nestes casos, portanto, não é possível correlacionar as elevações nos índices RDW com o VCM.

\section{Conclusões}

Com base no exposto, é possível observar que os valores de referência encontrados foram próximos aos preconizados na literatura, sem diferença entre os sexos. Além disso, é possível estabelecer uma nova divisão de faixas etárias para cada um dos índices estudados no presente trabalho, de acordo com os resultados estatísticos encontrados.

Dessa forma, para o VCM, as faixas etárias 7 a 10 anos e 11 a 17 anos podem ser consideradas como uma só em estudos posteriores, pois não apresentaram diferença significativa entre eles. O mesmo ocorre com o RDW-CV entre os grupos 18 a 59 anos e maiores de 60 anos, bem como com RDW-SD para os menores de 6 anos e os de idade entre 7 a 10 anos e com os de 11 a 17 anos e 18 a 59 anos.

Os estudos de correlação mostraram que o RDW-CV tem alta relação com o VCM microcítico, enquanto que o RDW-SD tem maior relação com o VCM macrocítico. Diferentemente, no grupo normocítico, constatou-se não ser possível estabelecer correlação entre VCM e os índices RDW, pois o mesmo apresentou alguns casos de anisocitose isolada, provavelmente devido à ocorrência de anisocitose precoce à anemia ou de outras doenças mencionadas anteriormente. Esses resultados confirmaram os encontrados pela maioria das publicações consultadas. 


\begin{abstract}
The term anisocytosis indicates the degree of variation in the size of red cells observed on the microscope. The above expression is observed in the automated hemogram through two parameters: RDW-CV Red Blood Cell Distribution Width measured by Variation Coeficient) and RDW-SD the same measured by Standard Deviation), which are calculated from the mean corpuscular volume $(M C V)$, which represents the average size of erythrocytes. Together, the two parameters RDW and MCV may help in the differential diagnosis of various diseases, particularly certain types of anemia. The objective of this study was to determine the reference values of the parameters $R D W$, and verify the existence of any relation between these and the MCV through the hemograms of 306 patients in the clinic of Central Laboratory of the Hospital Universitário Oswaldo Cruz. In addition it was also determined the reference values in relation to sex and age. Moreover, to verify the possible relation between the RDW parameters and MCV it was performed the statistical test of Pearson correlation. The reference values for the general population were similar to those found in the literature, whereas the comparison of results did not show significant differences between sexes. The study by age group showed the need to reassess the division of age groups in future studies with these parameters, because some of these groups showed no significant difference between them. The statistical results showed a correlation for the relationship between microcytic MCV and $R D W-C V$ and between the macrocytic $M C V$ and RDW-SD. For the VCM normocytic significant correlation was observed only with the RDW-SD. Rev. Bras. Hematol. Hemoter.
\end{abstract}

Key words: Blood; reference values; erytrocytes; erythrocytes index.

\section{Referências Bibliográficas}

1. Almeida S, Carvalho P, Salgado M. Importância clínica do RDW na criança com anemia. Saúde Infantil. 2006.28(2).p 7-11.

2. Failace R. Anemia: Generalidades. In:__. Hemograma: Manual de Interpretação. $4^{\mathrm{a}}$ Edição. Porto Alegre: Editora Artmed, 2003. p. 60-67.

3. Villas Boas MVC, Araújo FMO, Gilberti MFP, Grotto HZW. Utilidade do RDW-SD como auxiliar na interpretação das alterações morfológicas dos eritrócitos. Roche Diagnóstica 2003.

4. Nascimento ML. Por que na relação entre a concentração de hemoglobina globular média e a contagem de hemácias a avaliação do RDW-SD é importante? NewsLab 2003 - Edição 61, p.94-104.

5. Bain BJ. Valores de Referência. In: Células Sanguíneas: Um Guia Prático. $2^{\circ}$ Edição. Porto Alegre. Editora Artes Médicas. 1997. p. 160.

6. Almeida CAN, Ramos APP, João CA, João CR, Ricco RG, Dutra-deOliveira JE. Jardinópolis sem anemia, primeira fase: avaliação antropométrica e do estado nutricional de ferro. Rev Paul Pediatr 2007;25(3):254-7.

7. Silva CF, Vitalle MSS, Quaglia EC, Braga JAP, Medeiros EHGR. Proporção de anemia segundo estadiamento puberal segundo dois critérios diagnósticos. Rev. Nutr. 2007.20(3):297-306.

8. Macedo LA(2002). In Barbosa D L, Arruda IKG, Diniz A. Prevalência e caracterização da anemia em idosos do Programa de Saúde da Família. Rev. Bras. Hematol. Hemoter. 2006;28(4):288-92.
9. Costa AC, Ribeiro B, Costa E. Índices plaquetários em indivíduos com doença hepática alcoólica crônica. Arquivo Gastroenterol. 2007;44(3).

10. Proctor SJ, Cox JR, Sheridan TJ. Anisocytosis and the C-1000 Channelyzer in macrocytic anaemia. J Clin Pathol. 1976;29 (8):719-23.

11. Davidson RJ, Hamilton PJ. High mean red cell volume: Its incidence and significance in routine haematology. J Clin Pathol. 1978;31 (5):493-8.

12. Ferreira FC. Frequência de anemias através da interpretação do eritrograma por automação no Chagas Laboratório de Análises Clínicas em Ubaitaita - Bahia. Monografia para conclusão do curso de Especialização em Hematologia Clínica. 2004.

13. Matos JF, Carvalho MG, Dusse LMS, Ferreira MFR, Stubbert, RVB. O papel do RDW, da morfologia eritrocitária e de parâmetros plaquetários na diferenciação entre anemias microcíticas e hipocrômicas. Rev. Bras. Hematol. Hemoter. 2008;30(6):463-9.

14. Aslan D, Gumruk F, Gurgey A, Altay C. Importance of RDW value in differential diagnosis of hypochrome anemias. Am J Hematol. 2002;69(1):31-3.

15. Akai Y, Fubota F, Bichile SK, Mehta NB, Pangam PR, Parekh V, et al. A study of $\mathrm{b}$ thalassemia screening using an automated hematology analyzer. Sysmex Journal International 1998;8(2).

16. Melo MR, Purini MC, Cançado RD, Kooro F, Chiattone CS. Uso de índices hematimétricos no diagnóstico diferencial de anemias microcíticas: Uma abordagem a ser adotada? Rev Assoc Med Bras 2002;48(3):222-4.

17. Bain BJ. Desordens dos eritrócitos e das plaquetas. In: Células Sanguíneas: Um Guia Prático. $2^{\mathrm{a}}$ Edição. Porto Alegre. Editora Artes Médicas. 1997. p. 204-208.

Avaliação: Editor e dois revisores externos Conflito de interesse: sem conflito de interesse

Recebido: 01/04/09

Aceito após modificações: 27/05/2009 